\title{
Correspondence
}

\section{Two-Dimensional Block Transforms and Their Properties}

MAHMOOD R. AZIMI-SADJADI AND ROBERT A. KING

\begin{abstract}
For two-dimensional (2-D) digital filters implemented by a block recursive equation, explicit relations between their frequency characteristics and those of scalar filter are obtained. Specifically, these include the relation between the discrete-time Fourier transform (DTFT) of the block recursive equation and that of the scalar 2-D difference equation, and the relation between the block matrix transfer function of the block processor and the scalar transfer function. These relations that are independent of the type of realization of the block processor have been obtained using the eigenvalue properties of a special type of circulant matrix introduced in this correspondence.
\end{abstract}

\section{INTRODUCTION}

The idea of processing sequences in blocks arose in connection with the desire to accomplish recursive filtering operations using fast transform techniques [1]. Later, it was found that the block processing also exhibits several other prominent benefits such as reduced quantization effects and increased data throughput rate when implemented using array processors. Mitra and Gnanasekaran [2] have developed several new structures and obtained the relation between the pole locations of the one-dimensional (1-D) implemented filter and those of the original transfer function. More recently, Barnes and Shinnaka [3] have developed a block statespace structure from a single-input single-output (SISO) state-space structure and derived explicit relations between the $z$-transform and the matrix transfer function of their multiinput and multioutput (MIMO) structure and those of the SISO model.

Two-dimensional (2-D) block processing has attracted considerable attention over the past few years. Several authors have developed different 2-D block structures for filters described by difference equation [4]-[6], convolution summation [4]-[6], and statespace formulations [4]. Mertzios and Venetsanopoulos [7] have derived the matrix transfer function of the general 2-D block recursive structure and used it in conjunction with polynomial matrix decomposition to arrive at a parallel decomposed realization without delay-free loops.

In this correspondence, new explicit relationships between the frequency characteristics of the 2-D block implemented system and those of the original scalar system are derived. Specifically, these include the relation between the DTFT of the block recursive equation and that of the 2-D difference equation; and the relation between the block matrix transfer function and the original transfer function. For a 2-D block implemented nonrecursive digital filter, these relations reduce to the DFT operations. The proposed method exploits the eigenvalue properties of a special type of block circulant matrix introduced here and their correspondence with the DFT of a finite sequence defined on the primitive roots of a number other than unity.

Manuscript received June 26, 1985; revised July 22, 1986.

M. R. Azimi-Sadjadi is with the Department of Electrical Engineering, Colorado State University, Fort Collins, CO 80523.

R. A. King is with the Department of Electrical Engineering, Imperial College of Science and Technology, London SW7 2BT, England.

IEEE Log Number 8610881.

\section{Two-Dimensional DTfT of Blocked Sequences}

Consider a causal quarter-plane $2-\mathrm{D}$ recursive digital filter that is implemented by the following block equation [4]:

$$
\begin{aligned}
& C_{00} Y_{i, j}+C_{10} Y_{i-1, j}+C_{01} Y_{i, j-1}+C_{11} Y_{i-1, j-1} \\
& \quad=D_{00} X_{i, j}+D_{10} X_{i-1, j}+D_{01} X_{i, j-1}+D_{11} X_{i-1, j-1} .
\end{aligned}
$$

In this equation $X_{i, j}$ is the $(i, j)$ th input block of size $K \times L$ (greater than the order of the filter), arranged as a column vector, i.e.,

$$
X_{i, j}=\left[\hat{X}_{i K}^{(j)} \hat{X}_{i K+1}^{(j)} \cdots \hat{X}_{i K+K-1}^{(j)}\right]^{\dagger}
$$

where

$$
\hat{X}_{m}^{(j)}=\left[x_{m, j L} x_{m, j L+1} \cdots x_{m, j L+L-1}\right]
$$

and similarly for $Y_{i, j} .\left\{x_{i, j}\right\}$ and $\left\{y_{i, j}\right\}$ represent the scalar input and output sequences, respectively; matrices $C_{i j}$ 's and $D_{i j}$ 's are either lower or upper triangular block Toeplitz, in which the constituent blocks are also, either lower or upper triangular Toeplitz matrices, whose elements are the coefficients $a_{i, j}$ 's and $b_{i, j}$ 's of the scalar 2$\mathrm{D}$ difference equation [4].

The DTFT of the input block sequence $\left\{X_{i, j}\right\}$ and the output block sequence $\left\{Y_{i, j}\right\}$ are, respectively, defined by $\tilde{X}\left(\omega_{1}, \omega_{2}\right)$ and $\tilde{Y}\left(\omega_{1}, \omega_{2}\right)$ where

$$
\tilde{X}\left(\omega_{1}, \omega_{2}\right)=\sum_{i=0}^{\infty} \sum_{j=0}^{\infty} X_{i, j} e^{-j\left(\omega_{1} i+\omega_{2} j\right)} .
$$

Using the definition of $X_{i, j}$ in (2), (3) can alternatively be written as

$$
\begin{aligned}
\tilde{X}\left(\omega_{1}, \omega_{2}\right)= & {\left[X_{0,0}\left(\omega_{1}, \omega_{2}\right) \cdots X_{0, L-1}\left(\omega_{1}, \omega_{2}\right) X_{1,0}\left(\omega_{1}, \omega_{2}\right)\right.} \\
& \left.\cdots X_{1, L-1}\left(\omega_{1}, \omega_{2}\right) \cdots X_{K-1, L-1}\left(\omega_{1}, \omega_{2}\right)\right]^{t}
\end{aligned}
$$

where $X_{r, s}\left(\omega_{1}, \omega_{2}\right) ; r \in[0, K-1]$ and $s \in[0, L-1]$ is defined as

$$
X_{r, s}\left(\omega_{1}, \omega_{2}\right)=\sum_{i=0}^{\infty} \sum_{j=0}^{\infty} x_{i K+r, j L+s} e^{-j\left(\omega_{1} i+\omega_{2} j\right)} .
$$

The DTFT of the output block sequence can similarly be defined. Equation (5) represents the DTFT of the sampled version sequence sampled at the block rate. Equation (3) shows that the DTFT of the input block sequence $\left\{X_{i, j}\right\}$ is the DTFT of a vector sequence whose elements are the sampled version of the original input sequence taken at sampling intervals $K$ and $L$. The DTFT of the original sequence $X\left(\omega_{1}, \omega_{2}\right)$ can be recovered from the DTFT of the blocked sequence, i.e.,

$$
X\left(\omega_{1}, \omega_{2}\right)=\sum_{r=0}^{K-1} \sum_{s=0}^{L-1} X_{r, s}\left(\omega_{1} K, \omega_{2} L\right) e^{-j\left(\omega_{1} r+\omega_{2} s\right)} .
$$

Now taking the DTFT of both sides of (1) and using the shifty property yields

$$
\begin{aligned}
& \left(C_{00}+C_{10} e^{-j \omega_{1}}+C_{01} e^{-j \omega_{2}}+C_{11} e^{-j \omega_{1}} e^{-j \omega_{2}}\right) \tilde{Y}\left(\omega_{1}, \omega_{2}\right) \\
& \quad=\left(D_{00}+D_{10} e^{-j \omega_{1}}+D_{01} e^{-j \omega_{2}}+D_{11} e^{-j \omega_{1}} e^{-j \omega_{2}}\right) \tilde{X}\left(\omega_{1}, \omega_{2}\right)
\end{aligned}
$$

or alternatively,

$$
\tilde{C}\left(\omega_{1}, \omega_{2}\right) \tilde{Y}\left(\omega_{1}, \omega_{2}\right)=\tilde{D}\left(\omega_{1}, \omega_{2}\right) \tilde{X}\left(\omega_{1}, \omega_{2}\right) .
$$

Now since $C_{00}, C_{01}, C_{10}$, and $C_{11}$ are block Toeplitz matrices, it can be shown that $\tilde{C}\left(\omega_{1}, \omega_{2}\right)$ takes a special form of block circulant matrix in which the upper diagonal block elements are multiplied 
by $\Omega_{1} \triangleq e^{-j \omega_{1}}$. This type of block circulant will therefore be referred to as an $\left\{\Omega_{1}\right\}$-block circulant. In addition, each elemental block of $\tilde{C}\left(\omega_{1}, \omega_{2}\right)$ will also take a form of $\left\{\Omega_{2}\right\}$-circulant, where $\mathrm{\Omega}_{2} \triangleq e^{-j \omega 2}$. The following theorems are developed for these types of matrices.

Theorem 1: An $\{\Omega\}$-circulant is a circulant matrix in which the upper diagonal elements are multiplied by $\Omega$. The permutation matrix of order $K$, associated with this matrix, is defined by

$$
P(\Omega)=\left[\begin{array}{ccccc}
0 & 0 & 0 & \cdots & \Omega \\
1 & 0 & 0 & \cdots & 0 \\
0 & 1 & 0 & \cdots & 0 \\
( & \ddots & & \vdots & \vdots
\end{array}\right]_{K \times K}
$$

This permutation matrix can be diagonalized as

$$
\Pi(\Omega)=U_{K} P(\Omega) U_{K}^{-1}
$$

where

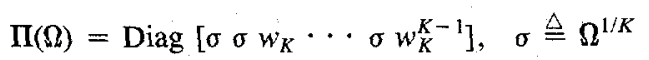

and

$$
w_{K}=\exp \left(\frac{-2 \pi j}{K}\right),
$$

and the columns of $U_{K}$ are the eigenvectors of $P(\Omega)$ corresponding to the relevant eigenvalues, $\sigma w_{K}^{i}$, therefore, we have

$$
U_{K}=W_{K} \Delta_{K}
$$

where

$$
\Delta_{K}=\operatorname{Diag}\left[1 \quad \sigma \sigma^{2} \cdots \sigma^{K-1}\right]
$$

and $W_{K}$ is a DFT matrix of size $K \times K$.

Theorem 2: The $\{\Omega\}$-circulant $C(\Omega)$ can be represented by its permutation matrix as

$$
\begin{aligned}
C(\Omega) & =\dot{c}_{0} I_{K}+c_{1} P(\Omega)+c_{2} P^{2}(\Omega)+\cdots+c_{K-1} P^{(K-1)}(\Omega) \\
& =\dot{R}[P(\Omega)]
\end{aligned}
$$

where $R[\cdot]$ is a scalar polynomial called a "representer" of the circulant. Matrix $C(\Omega)$ can be diagonalized as

$$
\begin{aligned}
\Lambda(\Omega) & =R[\Pi(\Omega)]=U_{K} C(\Omega) U_{K}^{-1} \\
& =\operatorname{Diag}\left[R(\sigma) R\left(\sigma w_{K}\right) \cdots R\left(\sigma w_{K}^{K-1}\right)\right] .
\end{aligned}
$$

In other words, if $\lambda_{i} ; i=0,1, \cdots, K-1$, are the eigenvalues of $P(\Omega)$ then the eigenvalues $\mu_{i}$ of $C(\Omega)$ are

$$
\mu_{i}=R\left(\lambda_{i}\right)=\sum_{m=0}^{K-1} c_{m} \lambda_{i}^{m}=\sum_{m=0}^{K-1} c_{m}\left(\sigma w_{K}^{i}\right)^{m} .
$$

Theorem 3: Consider matrix $\tilde{C}\left(\Omega_{1}, \Omega_{2}\right)$ which is an $\left\{\Omega_{1}\right\}$-block circulant of order $K L$, in which each constituent block is also an $\left\{\Omega_{2}\right\}$-circulant of order $L$. This matrix which is referred to as $\left\{\Omega_{1}\right.$, $\Omega_{2}$ \}-block circulant can be diagonalized as

$$
\tilde{\Lambda}\left(\Omega_{1}, \Omega_{2}\right)=\tilde{U}_{K, L} \tilde{C}\left(\Omega_{1}, \Omega_{2}\right) \tilde{U}_{K, \mathrm{~L}}^{-1}
$$

where

$$
\tilde{U}_{K, L}=U_{K} \otimes U_{L}
$$

and

$$
\tilde{\Lambda}\left(\Omega_{1}, \Omega_{2}\right)=\sum_{r=0}^{K-1}\left(\Pi^{r}\left(\Omega_{1}\right) \otimes \Lambda_{r}\left(\Omega_{2}\right)\right) .
$$

Matrices $\Pi^{r}\left(\Omega_{1}\right)$ and $\Lambda_{r}\left(\Omega_{2}\right)$ are defined by

$$
\Lambda_{r}\left(\Omega_{2}\right)=\operatorname{Diag}\left[\lambda_{r, 0}\left(\Omega_{2}\right) \lambda_{r, 1}\left(\Omega_{2}\right) \cdots \lambda_{r, L-1}\left(\Omega_{2}\right)\right]
$$

where

$$
\lambda_{r, l}\left(\Omega_{2}\right)=\sum_{s=0}^{L-1} c_{r, s}\left(\sigma w_{L}^{l}\right)^{s}, \quad \sigma \triangleq \Omega_{2}^{1 / L}, \quad l \in[0, L-1]
$$

and

$$
\Pi^{r}\left(\Omega_{1}\right)=U_{K} P^{r}\left(\Omega_{1}\right) U_{K}^{-1}
$$

where

$$
\Pi^{r}\left(\Omega_{1}\right)=\operatorname{Diag}\left[\varphi_{0}^{r}\left(\Omega_{1}\right) \varphi_{1}^{r}\left(\Omega_{1}\right) \cdots \varphi_{K-1}^{r}\left(\Omega_{1}\right)\right]
$$

and

$$
\varphi_{h}\left(\Omega_{1}\right)=\delta w_{K}^{h}, \quad \delta \triangleq \Omega_{1}^{1 / K}, \quad h \in[0, K-1] .
$$

Thús,

$$
\psi_{h, r}\left(\Omega_{1}\right)=\varphi_{h}^{r}\left(\Omega_{1}\right)=\left(\delta w_{K}^{h}\right)^{r} .
$$

Also,

$$
\tilde{U}_{K, L}=\left(W_{K} \Delta_{K}\right) \otimes\left(W_{L} \Delta_{L}\right)=\left(W_{K} \otimes W_{L}\right) \cdot\left(\Delta_{K} \otimes \Delta_{L}\right)
$$

where $W_{K}$ and $W_{L}$ represent the DFT matrices of size $K$ and $L$, respectively; and $\otimes$ denotes the Kronecker product of two matrices. Using theorem 3 and replacing $\Omega_{1} \triangleq e^{-j \omega_{1}}$ and $\Omega_{2} \triangleq e^{-j \omega_{2}}$, matrices $\tilde{C}\left(\omega_{1}, \omega_{2}\right)$ and $\tilde{D}\left(\omega_{1}, \omega_{2}\right)$ can be written as

$$
\begin{aligned}
& \tilde{C}\left(\omega_{1}, \omega_{2}\right)=\tilde{U}_{K, L}^{-1} \tilde{\Lambda}_{1}\left(\omega_{1}, \omega_{2}\right) \tilde{U}_{K, L} \\
& \tilde{D}\left(\omega_{1}, \omega_{2}\right)=\tilde{U}_{K, L}^{-1} \tilde{\Lambda}_{2}\left(\omega_{1}, \omega_{2}\right) \tilde{U}_{K, L}
\end{aligned}
$$

where $\tilde{\Lambda}_{1}$ and $\tilde{\Lambda}_{2}$ are diagonal matrices with elements defined by

$$
\begin{aligned}
\mu_{h, l}\left(\omega_{1}, \omega_{2}\right)= & \sum_{r=0}^{K-1} \sum_{s=0}^{L-1} \dot{a}_{r, s} \exp \left[-j\left(\frac{\omega_{1}+2 \pi h}{K}\right) r\right] \\
& \cdot \exp \left[-j\left(\frac{\omega_{2}+2 \pi l}{L}\right) s\right] \\
\eta_{h, l}\left(\omega_{1}, \omega_{2}\right)= & \sum_{r=0}^{K-1} \sum_{s=0}^{L-1} b_{r, s} \exp \left[-j\left(\frac{\omega_{1}+2 \pi h}{K}\right) r\right] \\
& \cdot \exp \left[-j\left(\frac{\omega_{2}+2 \pi l}{L}\right) s\right] .
\end{aligned}
$$

Now substituting (21) into (8) gives

$$
\begin{aligned}
\tilde{U}_{K, L} \tilde{Y}\left(\omega_{1}, \omega_{2}\right) & =\left[\tilde{\Lambda}_{1}^{-1}\left(\omega_{1}, \omega_{2}\right) \tilde{\Lambda}_{2}\left(\omega_{1}, \omega_{2}\right)\right] \tilde{U}_{K, L} \tilde{X}\left(\omega_{1}, \omega_{2}\right) \\
& =\tilde{\Gamma}\left(\omega_{1}, \omega_{2}\right) \tilde{U}_{K, L} \tilde{X}\left(\omega_{1}, \omega_{2}\right)
\end{aligned}
$$

where

$$
\begin{aligned}
\tilde{\Gamma}\left(\omega_{1}, \omega_{2}\right)= & \operatorname{Diag}\left[\gamma_{0,0}\left(\omega_{1}, \omega_{2}\right) \gamma_{0,1}\left(\omega_{1}, \omega_{2}\right) \cdots \gamma_{0, L-1}\left(\omega_{1}, \omega_{2}\right)\right. \\
& \left.\cdot \gamma_{1,0}\left(\omega_{1}, \omega_{2}\right) \cdots \gamma_{K-1, L-1}\left(\omega_{1}, \omega_{2}\right)\right]
\end{aligned}
$$

and

$$
\begin{array}{r}
\gamma_{h, l}\left(\omega_{1}, \omega_{2}\right)=\eta_{h, l}\left(\omega_{1}, \omega_{2}\right) / \mu_{h, l}\left(\omega_{1}, \omega_{2}\right), \quad h \in[0, K-1], \\
l \in[0, L-1]=H\left(\frac{\omega_{1}+2 \pi h}{K}, \frac{\omega_{2}+2 \pi l}{L}\right)
\end{array}
$$

where $H\left(\omega_{1}, \omega_{2}\right)$ is the original scalar transfer function. The block matrix transfer function is an $\left\{\omega_{1}, \omega_{2}\right\}$-block circulant and is given by

$$
\tilde{H}\left(\omega_{1}, \omega_{2}\right)=\tilde{U}_{K, L}^{-1} \tilde{\Gamma}\left(\omega_{1}, \omega_{2}\right) \tilde{U}_{K, L} .
$$

This shows that the eigenvalues of the block matrix transfer function are the scalar original transfer function evaluated at $\omega_{1}=\left(\omega_{1}\right.$ $+2 \pi h) / K$ and $\omega_{2}=\left(\omega_{2}+2 \pi l\right) / L$. Thus, the block matrix transfer function can be decomposed into a parallel bank of filters with each having the original scalar LTI transfer function followed by a sampling rate compressor [9] (at the block rate) and a modulator to provide the frequency shift of $(2 \pi h / K, 2 \pi l l L)$ for the $(h, l)$ th elements of the blocks. The elements of vector $\tilde{U}_{K, L} \tilde{X}\left(\omega_{1}, \omega_{2}\right)$ are given 
by

$X_{h, l}^{\prime}\left(\omega_{1}, \omega_{2}\right)=\sum_{r=0}^{K-1} \sum_{s=0}^{L-1} w_{K}^{r h} w_{L}^{s l} X_{r, s}\left(\omega_{1}, \omega_{2}\right) \exp \left[-j\left(\frac{\omega_{1} r}{K}+\frac{\omega_{2} s}{L}\right)\right]$.

Now using the definition of $X_{r, s}\left(\omega_{1}, \omega_{2}\right)$ in (5) and applying the index mapping of $p=i K+r, q=j L+s$ to (26), we obtain

$$
\begin{aligned}
X_{h, l}^{\prime}\left(\omega_{1}, \omega_{2}\right)= & \sum_{p=0}^{\infty} \sum_{q=0}^{\infty} x_{p, q} \exp \left[-j\left(\frac{\omega_{1}+2 \pi h}{K}\right) p\right] \\
& \cdot \exp \left[-j\left(\frac{\omega_{2}+2 \pi l}{L}\right) q\right] .
\end{aligned}
$$

A similar equation can be obtained for the elements of $\tilde{U}_{K, L} \tilde{Y}\left(\omega_{1}\right.$, $\omega_{2}$ ) vector. Equation (27) represents the DTFT of the original input sequence which is first decimated and then modulated. To recover the original scalar output sequence, the process consists of interpolating [9] all the channel signals back to their high sampling rate and demodulating them back to their original spectral locations. It can be shown that for nonrecursive digital filters (also recursive digital filters with impulse response which dies out fairly fast so that the effect of aliasing is negligible), the above DTFT relationships will become DFT relations, and hence, the operations can efficiently be carried out using 2-D decomposed DFT. Note that the input and output will be considered to be periodic with period $P$ and $Q(P, Q$ are the dimensions of the input array), and thus, the blocks will have a periodicity of $P / K$ and $Q / L$. In this case, the multiplication by matrices $\Delta_{K}$ and $\Delta_{L}$ would essentially have the same effect as multiplying the inner transforms by the relevant twiddle factors.

\section{ConClusion}

In this correspondence, expressions are derived which relate the DTFT of a general 2-D recursive block structure to those of the scalar difference equation. A new type of circulant matrix is introduced which possesses certain eigenvalue properties which are used to obtain the relations between the DTFT of the block implemented filter and those of the scalar implemented filter. The original scalar sequences can be recovered from the DTFT of the blocked sequences using a proper transformation. Moreover, it is shown that the block matrix transfer function can be implemented by a bank of parallel scalar original transfer functions, sampling rate compressors, and appropriate modulators. For 2-D nonrecursive filters and also stable $2-D$ recursive filters with fast decaying impulse responses, the operations can efficiently be accomplished using FFT algorithms.

\section{REFERENCES}

[1] C. S. Burrus, "Block realization of digital filters," IEEE Trans. Audio-Electroacoust., vol. AU-20, pp. 230-235, Oct. 1972.

[2] S. K. Mitra and R. Gnanasekaran, "Block implementation of recursive digital filters-New structures and properties," IEEE Trans. Circuits Syst., vol. CAS-25, pp. 200-207, Apr. 1973

[3] C. W. Barnes and S. Shinnaka, "Block-shift invariance and block implementation of discrete-time filters," IEEE Trans. Circuits Syst., vol. CAS-27, pp. 667-672, Aug. 1980.

[4] M. R. Azimi-Sadjadi and R. A. King, "Two-dimensional block processors-Structures and implementations," IEEE Trans. Circuits Syst., vol. CAS-33, pp. 42-50, Jan. 1986.

[5] S. K. Mitra and R. Gnanasekaran, "Block implementation of two-dimensional digital filters," J. Franklin Inst., vol. 316, pp. 299-316, Oct. 1983.

[6] B. G. Mertzios, "Block realization of 2-D IIR digital filters," Signal Processing, vol. 7, pp. 135-149, Oct. 1984.

[7] B. G. Mertzios and A. N. Venetsanopoulos, "Block realization of 2-D IIR digital filters based on the decomposition theorem," in 7 th
Euro. Conf. Circuit Theory Design, Prague, Czechoslovakia, Sept. 1985 , pp. 435-438.

[8] P. J. Davis, Circulant Matrices. New York: Wiley, 1979

[9] R. E. Crochiere and L. R. Rabiner, Multirate Digital Signal Processing. Englewood Cliffs, NJ: Prentice-Hall, 1983.

\section{On Using Cooccurrence Matrices to Detect Periodicities}

\author{
ANÍBAL R. FIGUEIRAS-VIDAL, JOSÉ M. PAEZ-BORRALLO, \\ AND RAMÓN GARCIA-GOMEZ
}

Abstract-Drawbacks and limitations in applying the $\chi^{2}$ independence hypothesis test parameter over the elements of cooccurrence matrices are discussed. The proper kind of measures to be applied, essentially of degree of concentration on the main diagonal, is justified. Some simple parameters that can be used in this direction are introduced, and their performances illustrated using simulations. The possibility of designing "ad hoc" tests is also suggested.

\section{INTRODUCTION}

The sample amplitude cooccurrence matrix series is one of the representations of a segment of a discrete, quantized signal that preserves information about it. These matrices have as elements

$$
\begin{gathered}
\Psi_{i j}\left(S_{n}, k\right)=\#\left\{\left(m_{1}, m_{2}\right) \mid n-M+1 \leq m_{1}, m_{2}\right. \\
\left.\leq n ; m_{2}-m_{1}=k ; \quad S_{n}\left(m_{1}\right)=i, S_{n}\left(m_{2}\right)=j\right\} \\
0 \leq i, j \leq Q-1
\end{gathered}
$$

where $S_{n}(m)$ is the $(n)$ segment of the quantized register $S(m)$, i.e., $S_{n}(m)$ equals $S(m)$ if $n-M-1 \leq m \leq n$, and 0 otherwise (we assume $0,1, \cdots, Q-1$ as quantizing levels without losing generality), and "\#" indicates the number of elements of the pairs $\left(m_{1}, m_{2}\right)$ defined in (1). It is also possible to introduce a normalized cooccurrence matrix series having the same elements normalized by $N=\Sigma_{i} \Sigma_{j} \Psi_{i j}\left(S_{n}, k\right)$.

These definitions correspond to joint histograms of sample value pairs; therefore, we preserve significant information about the signal when using the series $\Psi_{i j}\left(S_{n}, k\right),|k| \leq M$. In [1], classical representations for a segment of signal, such as energy and autocorrelation, are derived from these matrices (quantization effects are excluded).

It is clear, therefore, that this matrix series provides detailed information about the structure of the signal; in particular, (quasi-) periodicity can be measured using these matrices. Terzopoulos introduces in [1] a parameter for each matrix, based on the $\chi^{2}$ goodness-of-fit test applied to measure sample dependence, and estimates the pitch period of voice speech segments locating the maxima of the sequence constituted by these $\chi^{2}$ parameters.

Terzopoulos remarks in $[1$, p. 10] that the unconditional use of the $\chi^{2}$ parameter in detecting periodicities is a too-simplistic criterion, and he mentions one of the cases in which this fails: an odd-

Manuscript received March 3, 1986; revised June 24, 1986

A. R. Figueiras-Vidal and R. Garcia-Gomez are with the Department of Signal Theory and Processing, ETSI de Telecommunicación, Polytechnic Universidad de Madrid, Ciudad Universitaria, 28040 Madrid, Spain.

J. M. Paez-Borrallo is with the Department of Signal Theory and Processing, ETSI de Telecommunicación, Polytechnic Universidad, Ciudad Universitaria, 28040 Madrid, Spain, and Electrônica ENSA, San Rafael, 6/Pol. Ind. Alcobendas, Madrid, Spain.

IEEE Log Number 8611154. 